\title{
Did green debt instruments aid diversification during the COVID-19 pandemic?
}

\author{
Paresh Kumar Narayan ${ }^{1 *}$, Syed Aun R. Rizvi and Ali Sakti ${ }^{3}$
}

*Correspondence:

paresh.narayan2014@gmail.

com

${ }^{1}$ Monash University,

Melbourne, Australia

Full list of author information

is available at the end of the

article

\begin{abstract}
Faced with a persistent pandemic, investors are concerned about portfolio diversification. While the literature on COVID-19 has evolved impressively, limited work remains on diversification opportunities. We contribute to the literature by exploring the volatility and co-movement of different sovereign debt instruments, including green sukuk, sukuk, bond and Islamic and conventional equity indices for Indonesia. Our results consistently point towards increased asset co-movement and weak profitability during the pandemic. Interestingly, sukuk and green sukuk have a 14\% correlation with stocks, suggesting potential diversification prospects in times of extreme shocks.
\end{abstract}

Keywords: COVID-19, Green Sukuk, Indonesia Capital market

JEL Classification: G11, G15, G19

\section{Introduction}

Global crises, whether financial or economic, bring to attention the role of portfolio diversification. The literature on portfolio diversification in times of crises is rich and perceives debt instruments as attractive tools (see, inter alia, Boucher and Tokpavi 2019; Selmi et al. 2019; Skintzi 2019). The current COVID-19 pandemic, because it is more persistent than any previous crisis, has had a dynamic effect on the financial system. In other words, the effects have been experienced in stages. The initial stage, for instance, was one when the pandemic started and markets overreacted; then, with time, as more was understood about the pandemic, markets corrected their overreaction (Harjoto et al. 2021). In this regard, many studies have demonstrated how equity markets (see, inter alia, Haroon and Rizvi 2020a,b; Narayan 2020a; Sharma 2020) and energy markets (see, for instance, Iyke 2020; Polemis and Soursou 2020; Gil-Alana and Monge 2020) globally have reacted to the pandemic over time. Some studies have also explored the interaction between different asset classes: exchange rates (Narayan 2020b), oil and exchange rates (Devpura 2020), stocks and exchange rates (Prabheesh and Kumar 2021), stocks and bonds (Papadamou et al. 2020) and cryptocurrencies (Yousaf and Ali 2020; Shahzad et al. 2021). ${ }^{1}$

\footnotetext{
${ }^{1}$ For a literature survey on COVID-19, see Sha and Sharma (2020) and Sharma and Sha (2021).
} author(s) and the source, provide a link to the Creative Commons licence, and indicate if changes were made. The images or other third party material in this article are included in the article's Creative Commons licence, unless indicated otherwise in a credit line to the material. If material is not included in the article's Creative Commons licence and your intended use is not permitted by statutory regulation or exceeds the permitted use, you will need to obtain permission directly from the copyright holder. To view a copy of this licence, visit http:// creativecommons.org/licenses/by/4.0/. 
The literature alluded to above contains an important gap: limited studies have considered diversification prospects in light of the pandemic from a green investment ${ }^{2}$ point of view. In this study, we explore the potential diversification prospects. We argue that faced with the pandemic, investors are likely to behave consistent with the flight-toquality phenomena. In this pandemic situation, we perceive investors as treating market volatility and connectedness between sovereign debt and equity markets differently (that is, by considering the dynamic and persistent nature of the pandemic). When diversification prospects are evaluated, a multi-asset class model is suitable. We, therefore, consider an asset class that includes Islamic debt and equity markets. Over the years, Islamic finance has developed into a unique asset class; see Narayan and Phan (2019) for a survey. ${ }^{3}$

A relatively recent innovation in the debt market is the green debt instruments. An attractive feature of this instrument has been its use in financing renewable energy projects globally (see, inter alia, Tang and Zhang 2020; Banga 2019; Hachenberg and Schiereck 2018). However, the role of the debt market in an asset diversification portfolio needs further inquiry given the ramifications of the COVID-19 pandemic.

To explore the volatility and inter-connectedness of multiple asset classes within stocks and debt during the COVID-19 pandemic, we focus on the conventional stock market (IDX Composite/Indeks Harga Saham Gabungan), its Islamic counterpart (Jakarta Islamic Index), the conventional debt market (sovereign bond), its Islamic counterpart (sovereign sukuk), and the green sukuk from Indonesia. Our hypothesis is that the interplay between these asset classes has evolved and changed owing to the pandemic. Our hypothesis is motivated by earlier work on previous pandemics by Bhuyan et al. (2010), who highlight that stock market returns of the infected countries exhibit a significant increase in co-movements. The evolving recent literature shows that pandemic has impacted asset prices substantially differently during the COVID-19 period. Amongst these studies, Iyke (2020) shows not only that the pandemic predicts asset prices but how its influence changed during the pandemic. ${ }^{4}$ Focusing on the exchange rate market, Narayan (2020a) shows how bubble activity intensified, and how the exchange rate became more resilient to shocks during the pandemic. Moreover, Narayan (2020b) shows how the oil market return/volatility changed due to the pandemic. In addition, Prabheesh and Kumar (2021) show that exchange rate remains neutral while energy and financial markets were affected

\footnotetext{
${ }^{2}$ Green investments are investments in asset classes, which are aligned with a commitment to the promotion of environmentally friendly business practices and the conservation of natural resources. Green stocks are equities of environmentally friendly companies while green debt instruments are a type of fixed-income instrument that are specifically earmarked to raise money for climate and environmental project.

${ }^{3}$ Several studies on equities have explored the unique characteristics of Islamic equities (see, inter alia, Rizvi and Arshad 2018; Rana and Akhter 2015) and for debt instruments (Sukuk) (see, for instance, Azmat et al. 2017; Naifar and Hammoudeh 2016; Önder 2016).

4 Several studies on COVID-19 have shown how the pandemic has influenced and shaped economic and financial relationship between and within a country and amongst groups of countries such as those belonging to regions. For a partial list of studies, see Prabheesh (2020), who examines stock and foreign portfolio investments during pandemic for India; Gil-Alana and Claudio-Quiroga (2020) evaluate the response of Asian stock markets to the pandemic; Yan and Qian (2020) and He et al. (2020) evaluate the reaction of the Chinese stock market to the pandemic; Liu et al. (2020) test the oil-stock returns nexus for the US; Xu (2020) studies stock returns of USA and Canada; Djurovic et al. (2020) study affect on Montenegro, while Sergi et al. (2021) and Ashraf (2020) consider this relation for 76 and 43 countries, respectively; So et al. (2020) examine the case of Hong Kong stock returns; and Salisu et al. (2020) focus on the OECD countries. In related work, Haroon and Rizvi (2020b) show how financial market liquidity is impacted by the pandemic. See also Narayan, Devpura and Wang (2020) and Wei et al (2020) for the exchange rate-COVID-19 analysis.
} 
by COVID uncertainty. ${ }^{5}$ Other studies offer equally important insights: Appiah-Otoo (2020), for instance, reveal using Chinese data that exchange rate significantly reduces domestic credit during the pandemic; Salisu and Sikiru (2020) find that during the COVID-19 period uncertainty of the pandemic is a factor for Asia-Pacific Islamic stock returns; and Qin et al. (2020) show that the pandemic has a negative effect on the oil price. Overall, we conclude from the literature that the impact of the recent pandemic on financial markets has exacerbated uncertainty - a point demonstrated by Sharma (2020). This literature, therefore, inspires the following questions. What has happened to asset price correlations over time including over the pandemic period? Has this portfolio diversification or otherwise influenced profits from those asset classes we consider? Has there been a flight to quality because of the uncertainty created by the pandemic? Our hypothesis allows us to address these questions and contribute to our understanding of asset pricing behavior in the COVID-19 pandemic period.

Our focus on Indonesia has roots in its unique structure and diverse set of financial asset class offerings. As highlighted by Sharma et al. (2019), Indonesia is uniquely poised as its equity market is large yet underdeveloped, offering opportunities for growth. As the fourth largest global population, the Indonesian market boosts of a range of diverse products across both conventional and Islamic asset classes. Indonesia also boosts the largest Muslim population in the world offering a range of Islamic products. Indonesia is also a leader in sovereign issuance of green sukuk. ${ }^{6}$ These features make a study on Indonesia's asset co-movement from the point of view of diversification benefits ideal.

We test our hypothesis on the evolution of risks and returns from multiple two-asset portfolios by using daily data (March 4, 2019 to December 4, 2020) fitted to a multivariate GARCH model. To evaluate the impact of the COVID-19 pandemic, the sample is divided into a pre-COVID-19 and a COVID-19 sub-sample periods. Our findings and contributions are as follows. We show that the volatility of all assets increased during the COVID-19 pandemic. While this finding adds to the evolving literature on financial market volatility during COVID-19 (see Haroon and Rizvi 2020a, b; Ali et al. 2020; Salisu and Adediran 2020), the insights we provide come from a unique set of assets that include less riskier assets, such as sukuk and green sukuk, which have not been subjected to this type of empirical investigation before.

We, therefore, explore the correlations between asset pairs consisting of conventional and Islamic stocks, bonds, and sukuk and green sukuk. By considering a mixture of risky and less risky assets, we offer new insights on the dynamic relationship among assets that have implications for portfolio formation and risk diversification. We find high correlations: averaging over $80 \%$ between stocks, and these correlations increased during the pandemic. Both sukuk and green sukuk have low correlations with stocks and although these correlations increase in the pandemic period, they remain low, at less

\footnotetext{
${ }^{5}$ One of the most influential strands of the COVID-19 literature relates to the energy sector; see, for instance, Ertuğrul et al. (2020) who find evidence of a high volatility pattern of the Turkish diesel market during COVID-19. Polemis and Soursou (2020) find that the pandemic influenced stock returns of the Greek energy firms. Akhtaruzzaman et al. (2020) disclose COVID-19 as a moderator to the oil price shock. Gharib et al. (2020) find contagion effect of bubbles in oil and gold markets during the COVID-19 pandemic. Amar et al. (2020) show spillovers of commodity and stock prices in oil producing and consuming countries during the COVID-19 period.

${ }^{6}$ Green sukuk and bond initiative of the Indonesian government is part of efforts to reduce greenhouse gas emissions. Initiated in 2018, the green sukuk issuance raised $\$ 1.25$ billion to finance environment friendly infrastructural projects across Indonesia.
} 
than $14 \%$. The implication is that both sukuk and green sukuk offer diversification benefits during the pandemic. When we utilize a two-asset portfolio consisting of the same pairs of assets used to obtain correlations, we find, in general, that diversification offers greater profits from a two-asset portfolio weight optimization model. Our findings support the mixed evidence in the literature on green debt instruments. This literature (see, for instance, Nguyen et al. 2021; Reboredo et al. 2020) finds evidence of: (a) low correlations between green bonds and commodities; and (b) price spillover from fixed income to green bonds. We add to this by showing potential diversification benefits when it comes to sukuk and green sukuk.

Finally, because in our exercise, we have both risky assets (such as the conventional and Islamic stocks) and less risky assets (such as bonds, sukuk and green sukuk), this allows us to test the flight to quality hypothesis-the idea that during crises investors prefer holding less risky assets. If this is true, then we should expect to see a switch from risky assets to less risky assets. Using a Granger causality test, we find strong evidence supporting Granger causality from Islamic and conventional stocks to sukuk and green sukuk. The evidence is, as expected, much stronger during the COVID-19 pandemic period.

Lastly, the COVID-19 pandemic has instigated a rich literature on the effects of the pandemic on the financial system and asset prices (see, inter alia, Yan and Qian 2020; Sharma 2020; Iyke 2020; Sha and Sharma 2020; Sharma and Sha 2020). In this literature, none of these studies has yet analyzed how sukuk and green sukuk are connected to stocks and bonds from a portfolio diversification perspective. By doing so, we provide additional insights on how asset connectedness has evolved in light of the pandemic. In addition, our study contributes to the evolving literature on sovereign debt classes and equity nexus which concludes with mixed results (see Samour et al. 2020; Golab et al. 2018; Allegret et al. 2017).

We engage in robustness tests to confirm our key findings. Our attempt starts with sample splitting. We decompose the full sample into a pre-COVID pandemic sub-sample, an epidemic sub-sample (before the COVID-19 was declared a pandemic), and a pandemic sub-sample owing to the date on which the World Health Organization (WHO) declared COVID-19 a pandemic. We consistently find evidence of low correlations between sukuk/green sukuk vis-à-vis other assets. Secondly, when we localize the date of the start of COVID-19 to Indonesia and create sub-samples, which are Indonesia-specific, our main results remain insensitive. Thirdly as an alternative econometric model, we employ the exponential GARCH model to estimate volatility for our data sample. Results from this exercise do not change our story.

\section{Data and methodology}

Given that the COVID-19 pandemic is a recent crisis, the data are limited. For instance, the first confirmed case was reported on December 31, 2019. In this study, we use Indonesian data: namely, Indonesia's sovereign 5-year debt instruments (bond, sukuk and green sukuk). For equities, we use the benchmark conventional stock market index (IDX Composite/ Indeks Harga Saham Gabungan) and the Islamic index from the Jakarta stock exchange (Jakarta Islamic Index). 
The data spans March 4, 2019 to December 4, 2020, which gives us 225 and 240 observation (in terms of days) for pre and post the first COVID-19 reported case on December $31,2020 .^{7}$ We also segregate the COVID-19 period into a pre-COVID-19 (March 4, 2019 to December 30, 2019) sample. We create a sub-sample marked by the period when the WHO had not declared COVID-19 a pandemic; we refer to this as an epidemic phase (December 31, 2019 to March 10, 2020). Finally, we have a pandemic sub-sample covering the time from when the WHO declared COVID-19 as a pandemic (March 11, 2020 to December 4, 2020). In additional analysis, we categorize the pre-COVID-19 and post-COVID-19 timelines localized specifically to the Indonesian context given that the first reported case in Indonesia was on March 2, 2020. ${ }^{8}$ With this date, for Indonesia, we have a pre-COVID-19 period from March 4, 2019 to March 1, 2020 and a COVID-19 period from March 2, 2020 to December 4, 2020.

Daily returns for all the sample are calculated using the equation, $r_{t}=\ln \left(P_{t}\right)-\ln \left(P_{t-1}\right)$. Here, $r_{t}$ and $P_{t}$ denote daily returns and price at the business day $t$, respectively, and $\ln$ represents the natural log.

To study the volatility and correlations of the sovereign bond, sukuk, green sukuk, Islamic stocks, and conventional stocks, we employ a Multivariate Generalized Autoregressive Conditional Heteroscedastic-Dynamic Conditional Correlation (MGARCH-DCC) model proposed by Engle (2002) and Pesaran and Pesaran (2009). The MGARCH-DCC is suitable to obtain the variance and correlations between assets over time. This method is popular and widely used in applications, and can be stated as:

$$
r_{t}=\mu_{t}+\varepsilon_{t}
$$

where $\mu_{t}=\mathrm{E}\left[r_{t}\left|\Omega_{t-1}\right|\right], \quad \mu_{t} \mid \Omega_{t-1} \sim N\left(0, H_{t}\right), \quad H_{t}=D_{t} R_{t} D_{t}, \quad D_{t}=\operatorname{diag}\left\{\sqrt{h_{i i . t}}\right\}$, and $z_{t}=D_{t}^{-1} \varepsilon_{t}$. In this, $h_{i i . t}$ is the estimated conditional variance from the individual univariate GARCH model; $D_{t}$ is the diagonal matrix of conditional standard deviations; $R_{t}$ is the time-varying conditional correlation coefficient matrix of returns; and $z_{t}$ is the standardized residuals vector with mean zero and variance one. The dynamic correlation coefficient matrix of the DCC model can be specified further as per Hsu et al. (2008):

$$
R_{t}=\left(\operatorname{diag}\left(Q_{t}\right)^{-1 / 2} Q_{t}\left(\operatorname{diag}\left(Q_{t}\right)\right)^{-1 / 2}\right)
$$

where $\quad Q_{t}=\left(q_{i j, t}\right) \quad$ and $\quad\left(\operatorname{diag}\left(Q_{t}\right)\right)^{-1 / 2}=\left(\operatorname{diag} \frac{1}{\sqrt{q_{11, t}}}, \ldots, \frac{1}{\sqrt{q_{n n, t}}}\right) \quad$ with $q_{i j, t}=\bar{\rho}_{i j}+a\left(z_{i, t-1} z_{j, t-1}-\bar{\rho}_{i j}\right)+\beta\left(q_{i j, t-1}-\bar{\rho}_{i j}\right)$ in which $\bar{\rho}_{i j}$ is the unconditional correlations and the new time-varying conditional correlation coefficient is $\rho_{i j, t}=q_{i j, t} / \sqrt{q_{i i, t} q_{j j, t}}$.

\section{Analysis}

\section{Descriptive statistics}

Table 1 provides descriptive statistics of the five assets. We report average returns and standard deviation of the data across various sub-samples. Panel A provides the

\footnotetext{
${ }^{7}$ https://www.who.int/news/item/27-04-2020-who-timeline---covid-19

${ }^{8}$ https://covid19.who.int/region/searo/country/id
} 
Table 1 Descriptive statistics

\begin{tabular}{|c|c|c|c|c|c|c|c|c|c|}
\hline & & \multirow[b]{2}{*}{$\begin{array}{l}\text { Full } \\
\text { Sample }\end{array}$} & \multicolumn{2}{|l|}{ Panel A } & \multicolumn{3}{|l|}{ Panel B } & \multicolumn{2}{|l|}{$X$} \\
\hline & & & $\begin{array}{l}\text { Pre- } \\
\text { COVID }\end{array}$ & $\begin{array}{l}\text { COVID- } \\
\text { Time }\end{array}$ & $\begin{array}{l}\text { Pre- } \\
\text { COVID }\end{array}$ & Epidemic & Pandemic & $\begin{array}{l}\text { Pre-COVID } \\
\text { (Indonesia) }\end{array}$ & $\begin{array}{l}\text { COVID-Time } \\
\text { (Indonesia) }\end{array}$ \\
\hline \multirow[t]{2}{*}{ Bonds } & Mean & $0.0038 \%$ & $0.0065 \%$ & $0.0016 \%$ & $0.0065 \%$ & $0.0199 \%$ & $-0.0033 \%$ & $0.0066 \%$ & $0.0010 \%$ \\
\hline & St. Dev & 0.0011 & 0.0011 & 0.0010 & 0.0011 & 0.0010 & 0.0010 & 0.0011 & 0.0000 \\
\hline \multirow[t]{2}{*}{ Sukuk } & Mean & $0.0197 \%$ & $0.0197 \%$ & $0.0197 \%$ & $0.0197 \%$ & $0.0312 \%$ & $0.0165 \%$ & $0.0227 \%$ & $0.0165 \%$ \\
\hline & St. Dev & 0.0028 & 0.0016 & 0.0035 & 0.0016 & 0.0021 & 0.0038 & 0.0016 & 0.0002 \\
\hline \multirow{2}{*}{$\begin{array}{l}\text { Green } \\
\text { sukuk }\end{array}$} & Mean & $0.0208 \%$ & $0.0271 \%$ & $0.0155 \%$ & $0.0271 \%$ & $-0.0097 \%$ & $0.0223 \%$ & $0.0260 \%$ & $0.0154 \%$ \\
\hline & St. Dev & 0.0019 & 0.0009 & 0.0025 & 0.0009 & 0.0016 & 0.0027 & 0.0008 & 0.0002 \\
\hline \multirow{2}{*}{$\begin{array}{l}\text { Conven- } \\
\text { tional } \\
\text { stock }\end{array}$} & Mean & $-0.0236 \%$ & $-0.0123 \%$ & $-0.0331 \%$ & $-0.0123 \%$ & $-0.3859 \%$ & $0.0624 \%$ & $-0.0366 \%$ & $-0.0100 \%$ \\
\hline & St. Dev & 0.0130 & 0.0072 & 0.0164 & 0.0072 & 0.0139 & 0.0169 & 0.0072 & -0.0001 \\
\hline \multirow{2}{*}{$\begin{array}{l}\text { Islamic } \\
\text { stocks }\end{array}$} & Mean & $-0.0299 \%$ & $-0.0044 \%$ & $-0.0514 \%$ & $-0.0044 \%$ & $-0.4856 \%$ & $0.0662 \%$ & $-0.0398 \%$ & $-0.0195 \%$ \\
\hline & St. Dev & 0.0162 & 0.0100 & 0.0200 & 0.0100 & 0.0171 & 0.0206 & 0.0099 & -0.0002 \\
\hline
\end{tabular}

This table presents the mean and standard deviations of the daily bond, sukuk, green sukuk, conventional stock index and Islamic stock index returns from Indonesia. First column provides the average values for the full sample of data, from March 4, 2019 to December 4, 2020. Panel A provides descriptive statistics for the pre-COVID-19 period (March 4, 2019 to December 30, 2019) and the COVID-19 period (December 31, 2019 to December 4, 2020). Panel B presents descriptive statistics for the pre-COVID-19 period (March 4, 2019 to December 30, 2019), the epidemic phase of COVID-19 (December 31, 2019 to March 10, 2020) and the pandemic phase (March 11, 2020 to December 4, 2020). Panel C defines the preCOVID-19 and the COVID-19 periods specific to Indonesia, where the pre-COVID-19 phase is from March 4, 2019 to March 1, 2020 and the COVID-19 phase is from March 2, 2020 to December 4, 2020

descriptive statistics for the pre-COVID-19 period (March 4, 2019 to December 30, 2019) and the COVID-19 phase (December 31, 2019 to December 4, 2020). Panel B presents corresponding descriptive statistics for the pre-COVID period (March 4, 2019 to December 30, 2019), the epidemic phase (December 31, 2019 to March 10, 2020), and the pandemic phase (March 11, 2020 to December 4, 2020). Panel C defines preCOVID-19 and COVID-19 periods specifically for Indonesia based on the first reported COVID-19 case in Indonesia as discussed earlier. ${ }^{9}$ Consistent with recent studies (see those in Sha and Sharma (2020)(2020 and Narayan et al. (2020), for instance), returns in the pandemic period have been relatively low with higher variance.

The data suggest that the sovereign sukuk issued by the Government of Indonesia is least affected in terms of average returns during the pandemic. We also observe that in the case of green sukuk, there was a sharp decline in returns during the epidemic phase but returns recovered during the pandemic phase. The implication is that because asset price reaction to the different phases of COVID-19 has been heterogeneous (see Sharma and Sha 2020), the conditional correlations and volatilities are likely to be heterogeneous.

\section{Correlations}

To formally test this, we turn to Tables 2 and 3 where average conditional volatility and conditional correlations for different asset combinations and sub-samples are presented. From results described in Table 2, we observe that over the full sample of data across the five assets, Islamic stocks were most volatile followed by conventional stocks. Bonds were least volatile together with green sukuk. Panel A tells that except for bonds, which became least volatile in the COVID-19 period, all other assets were

\footnotetext{
${ }^{9}$ https://covid19.who.int/region/searo/country/id
} 
Table 2 Average sample volatility of assets

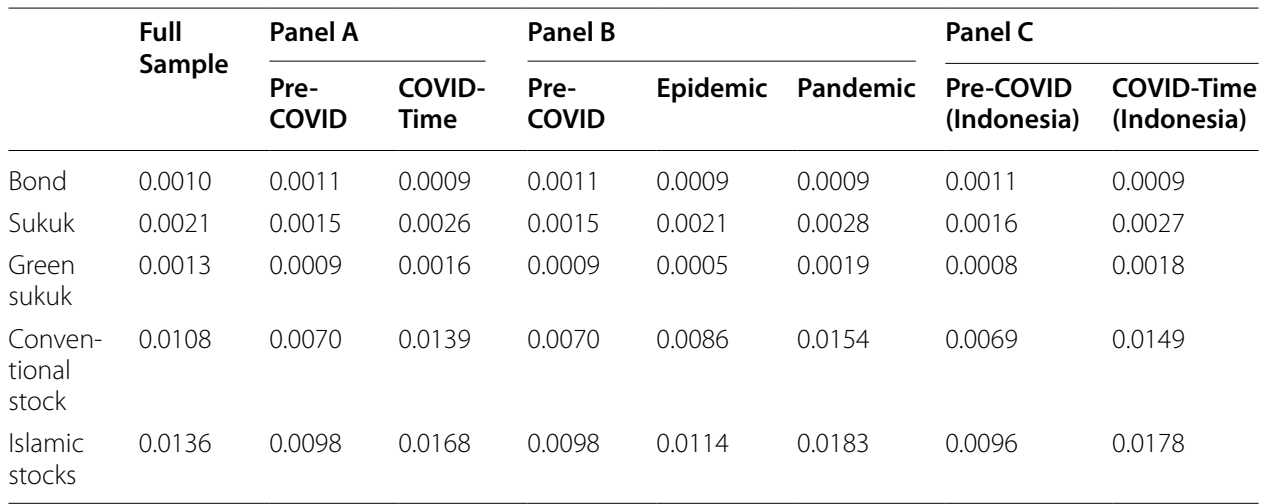

This Table provides the average of conditional volatilities of the five asset returns; namely bonds, sukuk, green sukuk, conventional stocks, and Islamic stocks. First column provides the average values for the full sample of data, from March 4, 2019 to December 4, 2020. Panel A provides descriptive statistics for the pre-COVID-19 period (March 4, 2019 to December 30, 2019) and the COVID-19 period (December 31, 2019 to December 4, 2020). Panel B presents descriptive statistics for the pre-COVID-19 period (March 4, 2019 to December 30, 2019), the epidemic phase of COVID-19 (December 31, 2019 to March 10, 2020) and the pandemic phase (March 11, 2020 to December 4, 2020). Panel C defines the pre-COVID-19 and the COVID19 periods specific to Indonesia, where the pre-COVID-19 phase is from March 4, 2019 to March 1, 2020 and the COVID-19 phase is from March 2, 2020 to December 4, 2020

more volatile in the COVID-19 period compared to the pre-COVID-19 period. This is true regardless of how we define COVID-19 dates (see Panel C). Panel B suggests that even when COVID-19 was not declared a pandemic - that is, at the epidemic stage, three of the five assets had higher volatility compared to the pre-COVID-19 period. In this regard, we see that bonds and green sukuk volatility was less sensitive to the epidemic. Overall, the largest increase in volatility is noted for stocks and least for sukuk-based assets.

Table 3 presents average conditional correlations. The correlations are divided into three parts. The first part of table provides conditional correlations for pairs of debt securities. The second part presents the conditional correlations of conventional stocks with bonds, sukuk, and green sukuk. The third part presents the conditional correlations of Islamic stocks with conventional stocks, bonds, sukuk and green sukuk. Starting with sukuk, green sukuk and bond correlations, we see that over the full sample, sukuk-green sukuk had the highest correlation at $39 \%$. In the preCOVID-19 period, it had the highest correlation at 51\%. In the COVID-19 phase, this correlation declined to around 30\% (Panel C). The sukuk-bond correlations were lowest, from around $14 \%$ in the full-sample to around $11 \%$ in the pre-COVID-19 sample (Panel C). It was the least at 3.54\% during the epidemic stage (Panel B). Overall, in the COVID-19 phase sukuk-bond correlations have increased compared to the preCOVID-19 phase. The exception is the correlation between green sukuk and sukuk, which declined in the COVID-19 phase.

The second part of the correlations between conventional stocks and bond/sukuk suggests that correlations have grown in the COVID-19 phase compared to the preCOVID-19 times. The weakest correlations though are for conventional stocks and sukuk followed by conventional stocks and green sukuk.

To the last set of results on Islamic stocks, conventional stocks, bonds, sukuk, and green sukuk, we see that stock level correlations are highest amongst all pairs and has 


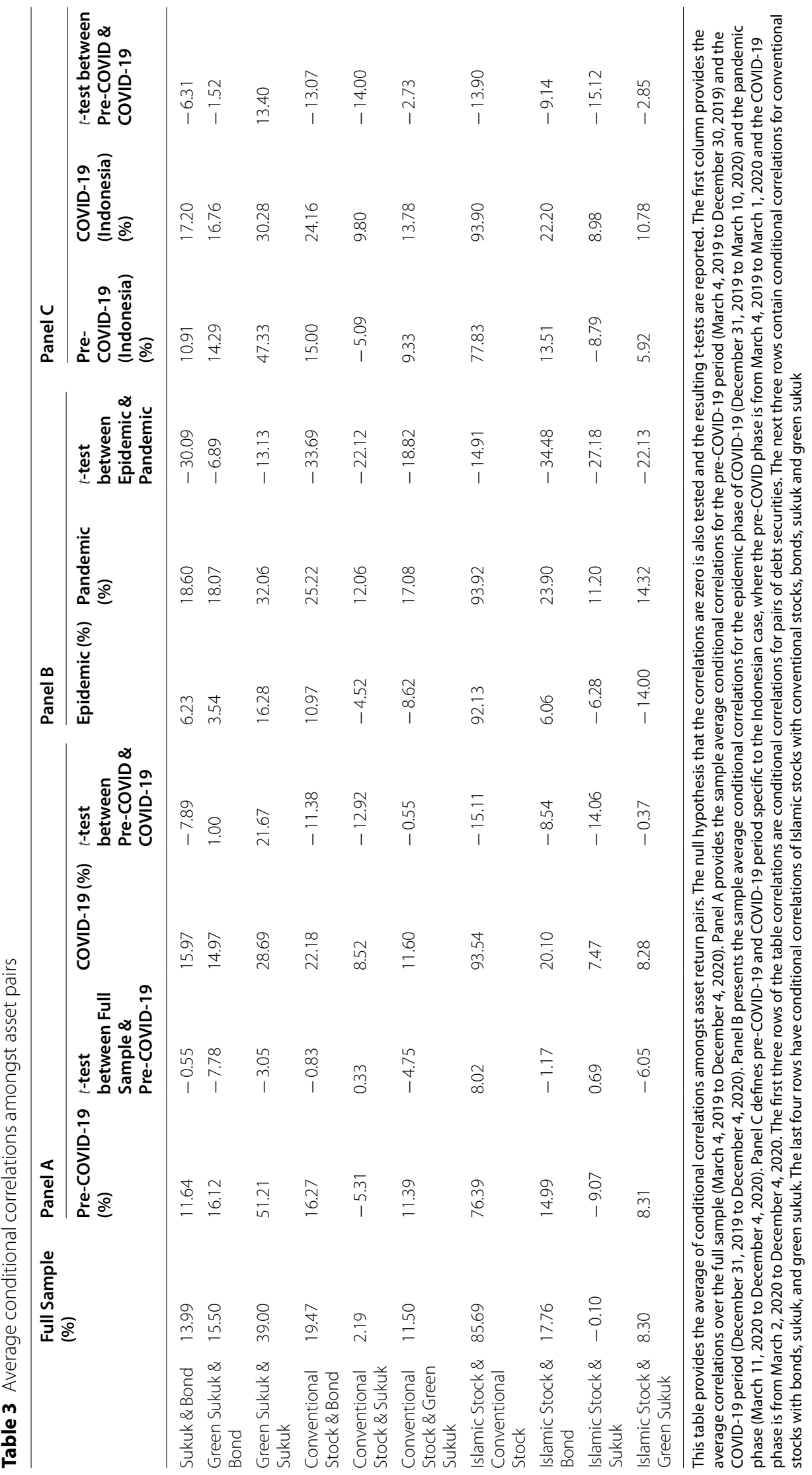


increased substantially over the COVID-19 period (average of over 93\%) compared to around $80 \%$ average correlation in the pre-COVID-19 period. Islamic stock-bond correlation has also increased in the COVID-19 period, but it is around 20\%. Islamic stocks and sukuk are weakly correlated both before and during the pandemic; however, in the COVID-19 phase, average correlation has risen to as much as $11 \%$. A similar pattern is observed for the correlation between Islamic stocks and green sukuk with correlations maximizing at $14 \%$ during the pandemic. This suggests flight-to quality phenomena, as discussed by Papadamou et al. (2020), regarding COVID-19's impact on financial asset classes.

\section{A test of the flight to quality hypothesis}

As we discussed in the introduction, our test of pair-wise correlations between asset classes was motivated by the flight to quality hypothesis: that investors prefer less risky assets during crises compared to riskier assets. One way of testing this would be examine if trading volume in risky assets, such as equity, increased during the COVID-19 pandemic. We are unable to test this given that trading volume data for those asset classes in our sample are unavailable. As an alternative, we employ a Granger causality test ${ }^{10}$ based on a VAR model with four lags. Our motivation for using a VAR model is as follows. If the riskier assets in our portfolio-namely, conventional and Islamic stocks-Granger cause less riskier assets-namely, bond, sukuk and/or green sukuk-this is evidence of flight to quality phenomena at work. To set the scene of how the assets have Granger caused each other, we start with results in Panel A of Table 4, based on the full sample of data. We see evidence of Granger causality running from conventional and Islamic stocks to green sukuk. When we explore the pre-COVID-19 flight to quality hypothesis with the COVID-19 phase, we find stronger evidence of flight to quality. That is, during the COVID-19 period, conventional and Islamic stocks Granger cause both sukuk and green sukuk. By comparison, in the pre-COVID-19 period, conventional and Islamic stocks only Granger caused green sukuk. Our work in this regard is preliminary and should be open to additional assessment of capital flight.

\section{Economic significance of correlations}

The objective of this sub-section is to explore, for each pair of our asset portfolio, profits based on obtaining a portfolio weight that is dynamic by construction. The idea is to see whether high asset correlations do indeed deliver less profits. In other words, the more diversified the portfolio (that is, the lower the correlations), the higher the profit. To achieve this goal, we draw on the two-asset portfolio weight optimization of Kroner and $\mathrm{Ng}$ (1998). To see how this weight is obtained, consider one of our pair of assets, namely Sukuk and bond. In this case, the weight $\left(w_{t}\right)$ of the Sukuk market in a one-dollar portfolio of Sukuk and bond (US) at time $t$ is given by:

${ }^{10}$ Granger Causality test has been used to understand and measure flight to quality in the literature where complete set of data is limited; see, for instance, Soylu, \& Güloğlu, (2019), and Corsi, Lillo, Pirino, \& Trapin (2018), \& Sarwar (2017). 


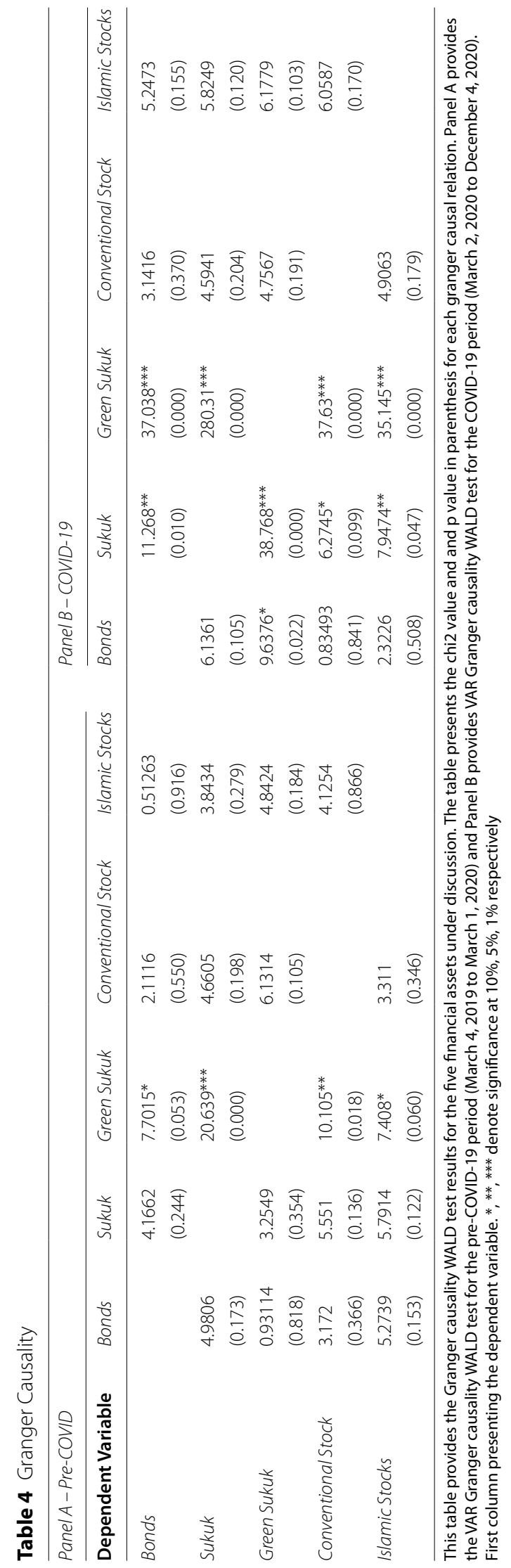


Table 5 Economic significance results

\begin{tabular}{lcc}
\hline & Pre-COVID-19 & COVID-19 \\
\hline Sukuk \& Bond & $5.95 \%$ & $2.59 \%$ \\
Green Sukuk \& Bond & $6.36 \%$ & $2.58 \%$ \\
Green Sukuk \& Sukuk & $8.88 \%$ & $6.16 \%$ \\
Conventional Stock \& Bond & $-9.61 \%$ & $6.26 \%$ \\
Conventional Stock \& Sukuk & $-5.67 \%$ & $9.80 \%$ \\
Conventional Stock \& Green Sukuk & $-6.38 \%$ & $9.37 \%$ \\
Islamic Stock \& Conventional Stock & $-24.75 \%$ & $14.66 \%$ \\
Islamic Stock \& Bond & $-12.39 \%$ & $8.24 \%$ \\
Islamic Stock \& Sukuk & $-12.27 \%$ & $11.76 \%$ \\
Islamic Stock \& Green Sukuk & $-9.08 \%$ & $12.37 \%$ \\
\hline
\end{tabular}

This table provides the optimized return which minimizes risk without reducing expected return as defined by Kroner and $\mathrm{Ng}$ (1998). The pre-COVID phase is from March 4, 2019 to March 1, 2020 and the COVID-19 phase is from March 2, 2020 to December 4, 2020

$$
w_{t}=\frac{h_{t}^{\text {Bond }}-h_{t}^{\text {Bond,Sukuk }}}{h_{t}^{\text {Sukuk }}-2 h_{t}^{\text {Sukuk,Bond }}+h_{t}^{\text {Bond }}}
$$

The time-varying conditional variance of the bond market $\left(h_{t}^{S u k u k}\right)$ and the bond market $\left(h_{t}^{\text {Bond }}\right)$, and the conditional covariance $\left(h_{t}^{\text {Sukuk,Bond }}\right)$ are extracted from estimating a bivariate GARCH model.

Two trends are worth a discussion: (1) we see that low-risk asset portfolios, in general, have lower profits (between 2 to 7\% in the COVID-19 pandemic period) compared to when one of the two assets in the portfolio is a riskier asset, such as the Islamic stockbond portfolio (8.24\%) and Islamic stock-sukuk portfolio (11.27\%); and (2) in general, profits are higher for portfolios of lower correlations (see Table 5).

A final observation is that the 10 -asset portfolios we consider are all profitable in the COVID-19 period, with annualized profits in the $2.59 \%$ (sukuk-bond) to $14.66 \%$ (conventional stock-Islamic stock) range. This is consistent with the stronger performance of the financial market in the aftermath of the early market panic due to the pandemic (see Sha and Sharma 2020 and Sharma and Sha 2020 for a summary of the literature).

\section{Robustness test}

One concern with our correlation and flight to quality hypothesis is that in taking returns we have not adjusted for other potential risk factors. We do this now. We proceed in two steps. In the first step we regress excess returns (on each asset) on excess market returns, stock price volatility, exchange rate, and day-of-the-week dummies variables. For further details, see notes to Table 6 where the results for the flight to capital hypothesis is reported. We see that our main finding is insensitive to the adjustment of potential market and macro risk factors. The flight to capital holds strongly during the COVID-19 phase. Not only Islamic (conventional) stocks Granger cause bonds and sukuk (bonds and green sukuk) but bonds and sukuk Granger cause green sukuk. 


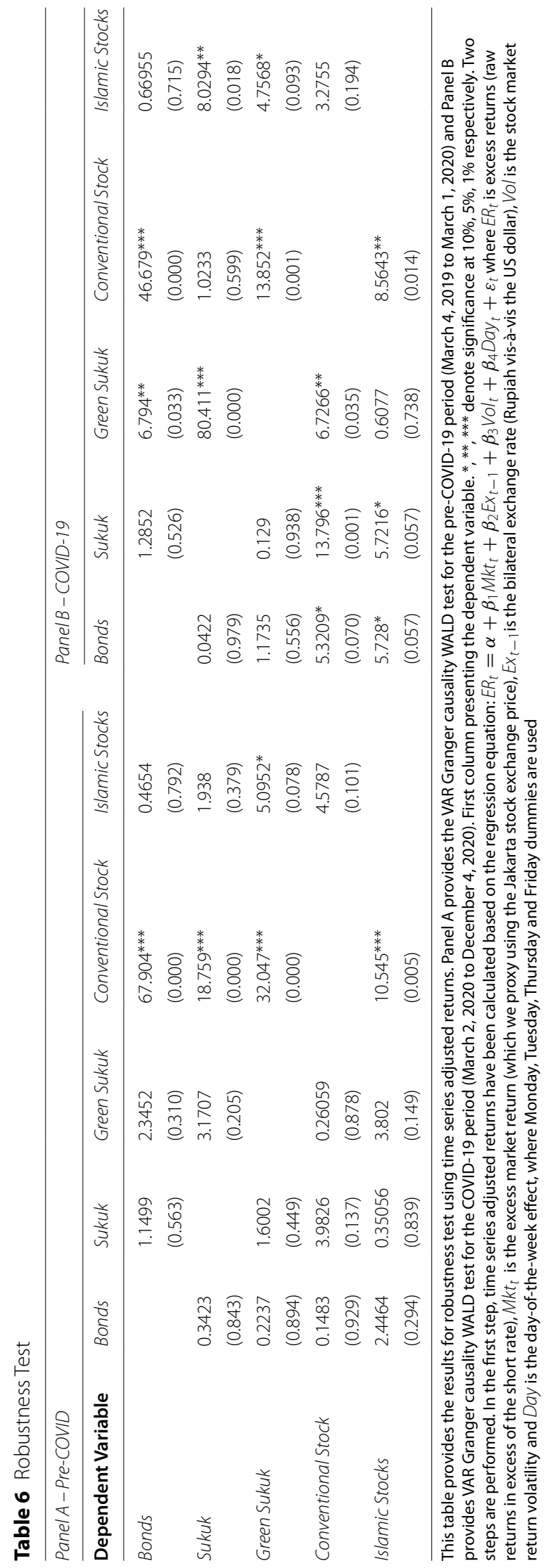




\section{Concluding remarks}

The COVID-19 pandemic has brought to attention the issue of portfolio diversification. This is also an issue for emerging markets such as Indonesia. In this paper, we focus on Indonesia given its progress on sukuk and green sukuk as financial instruments. We explore the volatility and correlation patterns in Indonesia's sovereign bond, its stock market (both conventional and Islamic markets), sukuk and green sukuk. In evaluating these five asset classes, we show that their volatility has not only increased over time but was also higher during the COVID-19 pandemic. Dynamic unconditional correlation analysis shows that correlations between asset pairs increased during the pandemic, and optimized portfolio weights (in a two-asset portfolio) offer less returns on average when correlations are high. We also show evidence of flight to quality, with investors switching from riskier assets (such as conventional and Islamic stocks) to less risky assets (such as sukuk and green sukuk). This evidence is immensely strong during the COVID-19 pandemic phase.

Our work suggests multiple directions for future research. First, asset correlations have clearly changed during the pandemic phase. It will be interesting to explore how these correlations have implications for dynamic trading strategies such as those of a mean-variance investor who tracks forecasted returns given an information set. It is also clear that the information set used to predict asset prices has also been influenced by the pandemic. Second, we have used a Granger causality test to deduce evidence of flight to quality. Future research should build on this by exploring other approaches and methods to exploring flight to quality in light of the pandemic.

\section{Author details}

${ }^{1}$ Monash University, Melbourne, Australia. ${ }^{2}$ Lahore University of Management Sciences, Lahore, Pakistan. ${ }^{3}$ Bank Indonesia, Jakarta, Indonesia.

Received: 12 February 2021 Accepted: 31 December 2021

Published online: 03 March 2022

\section{References}

Akhtaruzzaman M, Boubaker S, Chiah M, Zhong A (2021) COVID-19 and oil price risk exposure. Financ Res Lett 42:101882

Ali MH, Uddin MA, Khan MAR, Goud B (2020) Faith-based versus value-based finance: is there any portfolio diversification benefit between responsible and Islamic finance? Int J Financ Econ (In Press)

Allegret JP, Raymond H, Rharrabti H (2017) The impact of the European sovereign debt crisis on banks stocks. Some evidence of shift contagion in Europe. J Bank Finance 74:24-37

Amar AB, Belaid F, Youssef AB, Chiao B, Guesmi K (2021) The unprecedented reaction of equity and commodity markets to COVID-19. Financ Res Lett 38:101853

Appiah-Otoo I (2020) Does COVID-19 affect domestic credit? Aggregate and Bank level evidence from China. Asian Econ Lett 1(3):18074. https://doi.org/10.46557/001c.18074

Ashraf BN (2020) Economic impact of government interventions during the COVID-19 pandemic: international evidence from financial markets. J Behav Exp Finance 27:100371

Azmat S, Skully M, Brown K (2017) The (little) difference that makes all the difference between Islamic and conventional bonds. Pac Basin Financ J 42:46-59

Banga J (2019) The green bond market: a potential source of climate finance for developing countries. J Sustain Fin Invest 9:17-32

Bhuyan R, Lin EC, Ricci PF (2010) Asian stock markets and the severe acute respiratory syndrome (SARS) epidemic: implications for health risk management. Int J Environ Health 4(1):40-56

Boucher C, Tokpavi S (2019) Stocks and bonds: flight-to-safety forever? J Int Money Financ 95:27-43

Corsi F, Lillo F, Pirino D, Trapin L (2018) Measuring the propagation of financial distress with granger-causality tail risk networks. J Financ Stab 38:18-36

Devpura N (2020) Can oil prices predict Japanese yen. Asian Econ Lett. https://doi.org/10.46557/001c.17964

Djurovic G, Djurovic V, Bojaj MM (2020) The macroeconomic effects of COVID-19 in Montenegro: a Bayesian VARX approach. Financ Innov 6(1):1-16 
Engle R (2002) Dynamic conditional correlation: a simple class of multivariate generalized autoregressive conditional heteroskedasticity models. J Bus Econ Stat 20(3):339-350

Ertuğrul HM, Güngör BO, Soytaş U (2020) The effect of the COVID-19 outbreak on the Turkish diesel consumption volatility dynamics. Energy Res Lett. https://doi.org/10.46557/001c.17496

Gharib C, Mefteh-Wali S, Jabeur SB (2021) The bubble contagion effect of COVID-19 outbreak: evidence from crude oil and gold markets. Financ Res Lett 38:101703

Gil-Alana LA, Claudio-Quiroga G (2020) The COVID-19 impact on the ASIAN stock markets. Asian Econ Lett. https://doi. org/10.46557/001c.17656

Gil-Alana LA, Monge M (2020) Crude oil prices and COVID-19: persistence of the shock. Energy Res Lett 1(1):13200. https://doi.org/10.46557/001c.13200

Golab A, Jie F, Powell R, Zamojska A (2018) Cointegration between the European union and the selected global markets following sovereign debt crisis. Invest Manag Financ Innov 15(1):35-45

Hachenberg B, Schiereck D (2018) Are green bonds priced differently from conventional bonds? J Asset Manag 19:371-383

Harjoto MA, Rossi F, Lee R, Sergi BS (2021) How do equity markets react to COVID-19? Evidence from emerging and developed countries. J Econ Bus 115:105966

Haroon O, Rizvi SAR (2020a) COVID-19: media coverage and financial markets behavior-a sectoral inquiry. J Behav Exp Financ 27:100343

Haroon O, Rizvi SAR (2020b) Flatten the curve and stock market liquidity-an inquiry into emerging economies. Emerg Mark Financ Trade 56(10):2151-2161

Hsu Ku, Y. H., \&Wang, J. J. (2008) Estimating portfolio value-at-risk via dynamic conditional correlation MGARCH modelan empirical study on foreign exchange rates. Appl Econ Lett 15(7):533-538

lyke B (2020) COVID-19: the reaction of US oil and gas producers to the pandemic. Energy Res Lett 1(2):13912. https://doi. org/10.46557/001c.13912

Kroner KF, Ng VK (1998) Modelling asymmetric comovement of asset returns. Rev Financ Stud 11:817-844

Liu D, Sun W, Zhang X (2020) Is the Chinese economy well positioned to fight the COVID-19 pandemic? The financial cycle perspective. Emerg Mark Financ Trade 56(10):2259-2276

Naifar N, Hammoudeh S (2016) Do global financial distress and uncertainties impact GCC and global sukuk return dynamics? Pac Basin Financ J 39:57-69

Narayan PK, Phan DHB (2019) A survey of Islamic banking and finance literature: issues, challenges and future directions. Pac Basin Financ J 53:484-496

Narayan PK (2020a) Did bubble activity intensify during COVID-19? Asian Econ Lett. https://doi.org/10.46557/001c.17654

Narayan PK (2020b) Has COVID-19 changed exchange rate resistance to shocks? Asian Econ Lett. https://doi.org/10. 46557/001c.17389

Narayan PK, Devpura N, Wang H (2020) Japanese currency and stock market—What happened during the COVID-19 pandemic? Econ Anal Pol 68:191-198

Nguyen TTH, Naeem MA, Balli F, Balli HO, Vo XV (2021) Time-frequency comovement among green bonds, stocks, commodities, clean energy, and conventional bonds. Financ Res Lett 40:101739

Önder YK (2016) Asset backed contracts and sovereign risk. J Econ Behav Organ 132:237-252

Papadamou S, Fassas AP, Kenourgios D, Dimitriou D (2021) Flight-to-quality between global stock and bond markets in the covid era. Financ Res Lett 38:101852

Pesaran B, Pesaran MH (2009) Time series econometrics: using Microfit 5.0 (No. 330.015195 P48.). Oxford University Press, Oxford

Polemis M, Soursou S (2020) Assessing the impact of the COVID-19 pandemic on the Greek energy firms: an event study analysis. Energy Res Lett 1:3. https://doi.org/10.46557/001c.17238

Prabheesh KP (2020) Dynamics of foreign portfolio investment and stock market returns during the COVID-19 pandemic: evidence from India. Asian Econ Lett. https://doi.org/10.46557/001c.17658

Prabheesh KP, Kumar S (2021) The dynamics of oil prices exchange rates and the stock market under COVID-19 uncertainty: evidence from India. Energy Res Lett. https://doi.org/10.46557/001c.27015

Qin M, Zhang YC, Su CW (2020) The essential role of pandemics: a fresh insight into the oil market. Energy Res Lett 1(1):13166. https://doi.org/10.46557/001c.13166

Rana ME, Akhter W (2015) Performance of Islamic and conventional stock indices: empirical evidence from an emerging economy. Financ Innov 1(1):1-17

Reboredo JC, Ugolini A, Aiube FAL (2020) Network connectedness of green bonds and asset classes. Energy Econ 86:104629

Rizvi SAR, Arshad S (2018) Understanding time-varying systematic risks in Islamic and conventional sectoral indices. Econ Model 70:561-570

Salisu AA, Sikiru AA (2020) Pandemics and the Asia-Pacific Islamic stocks. Asian Econ Lett. https://doi.org/10.46557/001c. 17413

Salisu A, Adediran I (2020) Uncertainty due to infectious diseases and energy market volatility. Energy Res Lett. https:// doi.org/10.46557/001c.14185

Salisu AA, Akanni L, Raheem I (2020) The COVID-19 global fear index and the predictability of commodity price returns. $J$ Behav Exp Financ 27:100383

Samour A, Isiksal AZ, Gunsel Resatoglu N (2020) The impact of external sovereign debt and the transmission effect of the US interest rate on Turkey's equity market. J Int Trade Econ Dev 29(3):319-333

Sarwar G (2017) Examining the flight-to-safety with the implied volatilities. Financ Res Lett 20:118-124

Selmi R, Gupta R, Kollias C, Papadamou S (2019) The stock-bond nexus and investors'behavior in mature and emerging markets. Stud Econ Financ 38(3):562-582

Sergi BS, Harjoto MA, Rossi F, Lee R (2021) Do stock markets love misery evidence from the COVID-19. Finance Res Lett 42:101923 
Sha Y, Sharma S (2020) Research on pandemics special issue of the journal emerging markets finance and trade. Emerg Markets Finance Trade 56(10):2133-2137

Shahzad SJH, Bouri E, Kang SH, Saeed T (2021) Regime specific spillover across cryptocurrencies and the role of COVID19. Financ Innov 7(1):1-24

Sharma SS (2020) A note on the Asian market volatility during the COVID-19 pandemic. Asian Econ Lett. https://doi.org/ 10.46557/001c.17661

Sharma SS, Narayan PK, Thuraisamy K, Laila N (2019) Is Indonesia's stock market different when it comes to predictability? Emerg Markets Rev 40:100623

Sharma S, Sha Y (2020) Part A: special section on COVID-19 research. Emerg Mark Financ Trade 56(15):3551-3553. https:// doi.org/10.1080/1540496X.2020.1858617

Skintzi VD (2019) Determinants of stock-bond market comovement in the Eurozone under model uncertainty. Int Rev Financ Anal 61:20-28

So MK, Tiwari A, Chu AM, Tsang JT, Chan JN (2020) Visualising COVID-19 pandemic risk through network connectedness. Int J Infect Dis (In Press)

Soylu PK, Güloğlu B (2019) Financial contagion and flight to quality between emerging markets and US bond market. North Am J Econ Financ 50:100992

Tang DY, Zhang Y (2020) Do shareholders benefit from green bonds? J Corp Financ 61:101427

Wei Z, Luo Y, Huang Z, Guo K (2020) Spillover effects of RMB exchange rate among B\&R countries: before and during COVID-19 event. Financ Res Lett 37:101782

Xu D (2020) Canadian Stock Market Volatility under COVID-19 (No. 2001). University of Waterloo, Department of Economics

Yan L, Qian Y (2020) The impact of COVID-19 on the Chinese stock market: an event study based on the consumer industry. Asian Econ Lett. https://doi.org/10.46557/001c.18068

Yousaf I, Ali S (2020) Discovering interlinkages between major cryptocurrencies using high-frequency data: new evidence from COVID-19 pandemic. Financ Innov 6(1):1-18

\section{Publisher's Note}

Springer Nature remains neutral with regard to jurisdictional claims in published maps and institutional affiliations.

\section{Submit your manuscript to a SpringerOpen ${ }^{\circ}$ journal and benefit from:}

- Convenient online submission

Rigorous peer review

- Open access: articles freely available online

- High visibility within the field

Retaining the copyright to your article

Submit your next manuscript at $\boldsymbol{\Delta}$ springeropen.com 